01

\title{
Седиментация малоконцентрированной взвести стоксовских частиц в перемешиваемом слое с движущейся свободной границей
}

\author{
(C) А.В. Ряжских
}

Воронежский государственный технический университет, 394026 Воронеж, Россия

e-mail: ryazhskihav@bk.ru

Поступило в Редакцию 18 ноября 2018 г.

В окончательной редакции 18 ноября 2018 r.

Принято к публикации 11 марта 2019 г.

С использованием диффузионно-кинетической модели движения дисперсной фазы во взвесях рассмотрена задача о седиментации полидисперсных малоконцентрированных стоксовских частиц в условиях перемешивания несущей среды в плоском слое с движущейся свободной поверхностью.

Ключевые слова: седиментация, стоксовские частицы, осаждение, движущаяся граница.

DOI: $10.21883 /$ JTF.2019.08.47884.400-18

\section{Введение}

Прогнозирование изменения локальных характеристик твердой фазы в суспензиях с движущейся свободной границей дисперсионной среды является важным при описании различных процессов, таких как увеличение концентрации дисперсной фазы при концентрировании выпариванием [1], фильтровании суспензий [2], при проведении технологической операции перелива криожидкостей, содержащих кристаллические микропримеси [3] и др.

Применяемые формулировки начально-краевых задач с перемещаемыми свободными границами и методы их анализа $[4,5]$ в подавляющем большинстве случаев используются только для однородных сред с фазовыми превращениями на самой границе.

Однако и синтез математических моделей в рамках механики гетерогенных сред $[6,7]$ не приводит к желаемым результатам из-за трудностей, связанных с проблемами замыкания получаемых математических объектов, обладающих существенной нелинейностью, размерностью высокого порядка и т. п.

В связи с этим перспективным является использование диффузионно-кинетической модели, описывающей явления переноса дисперсной фазы во взвесях [8,9], которая подтвердила свою эффективность при решении различных предметно-ориентированных задач [10-13].

\section{1. Монодисперсная взвесь}

\section{1. Постановка задачи}

Предположим, что в начальный момент времени свободная граница дисперсионной среды в плоском слое взвеси монодисперсной твердой фазы стоксовских частиц в условиях перемешивания перемещается с некоторой постоянной скоростью в направлении дна, поверхность которого обладает свойством пропускать только несущую среду. В отсутствии скольжения фаз и, применяя одномерное нестационарное уравнение конвективно-диффузионной модели, запишем [14]:

$$
\begin{gathered}
\frac{\partial N(X, \theta)}{\partial \theta}=\frac{\partial N(X, \theta)}{\partial X}+\frac{1}{B o} \frac{\partial^{2} N(X, \theta)}{\partial X^{2}}, \\
N(X, \theta)=1, \\
(1-K) N(0, \theta)+\frac{1}{B o} \frac{\partial N(0, \theta)}{\partial X}=0, \\
N[H(\theta), \theta]+\frac{1}{B o} \frac{\partial N[H(\theta), \theta]}{\partial X}=0, \\
H(\theta)=1-\alpha \theta,
\end{gathered}
$$

где $X=x / h_{0}, N(X, \theta)=n(x, \tau) / n_{0}, H(\theta)=h(\tau) / h_{0}, \theta=$ $=\tau(w+v) / h_{0}, \quad K=k /(w+v), \quad \alpha=v /(w+v), \quad$ Bo $=$ $=(w+v) / D-$ число Боденштейна, $v-$ скорость движения свободной поверхности взвеси в направлении поверхности осаждения $[\mathrm{m} / \mathrm{s}], w-$ скорость осаждения частиц по Стоксу $[\mathrm{m} / \mathrm{s}], \tau-$ время $[\mathrm{s}]$; $D$ - коэффициент перемешивания частиц взвеси $\left[\mathrm{m}^{2} / \mathrm{s}\right]$, $h_{0}, h(\tau)$ - исходная и текущая высота слоя взвеси $[\mathrm{m}], n(x, \tau), n_{0}$ - локальная и начальная счетные концентрации частиц взвеси $\left[\mathrm{m}^{-3}\right], k-$ кинетический коэффициент, характеризующий скорость встраивания частиц размера $l$ в структуру осадка $[\mathrm{m} / \mathrm{s}], x-$ координата, направленная перпендикулярно поверхности осаждения в направлении свободной поверхности $[\mathrm{m}]$.

\section{2. Метод решения}

Пусть $H(\theta)=H$ является параметром, тогда начально-краевая задача (1)-(5) может быть решена с исполь- 
зованием преобразования Лапласа [15] по переменной $\theta$ :

$$
\begin{aligned}
& N(X, \theta)= \\
& \left\{\begin{array}{l}
N_{\mu}(X, \theta), \text { при } 4 K /[(1-2 K) B o]>H ; \\
N_{\lambda}(X, \theta)+N_{\mu}(X, \theta), \text { при } K<1 / 2 \text { и } 4 K /[(1-2 K) B o] \leq H,
\end{array}\right.
\end{aligned}
$$

где

$$
\begin{aligned}
N_{\mu}(X, \theta)= & \sum_{n=1}^{\infty}\left[A_{\mu} \frac{\sin \left(\mu_{n} X\right)}{\mu_{n}}+B_{\mu} \cos \left(\mu_{n} X\right)\right] \\
& \times \exp \left[-\left(\frac{B o}{4}+\frac{\mu_{n}^{2}}{B o}\right)-\frac{B o}{2} X\right] / C_{\mu} ; \\
A_{\mu}=- & (1-K)\left[\frac{1}{2} \cos \left(\mu_{n} H\right)-\frac{\mu_{n}}{B o} \sin \left(\mu_{n} H\right)\right] \\
+ & \left(\frac{1}{2}-K\right) \exp \left(\frac{B o}{2} H\right) ;
\end{aligned}
$$

где $\mu_{n}$ - корни уравнения

$$
\begin{aligned}
& \operatorname{tg}\left(\mu_{n} H\right)=\frac{4 B o K \mu_{n}}{(1-2 K) B o^{2}+4 \mu_{n}^{2}} ; \\
& N_{\lambda}(X, \theta)=\left[A_{\lambda} \frac{\operatorname{sh}(\lambda X)}{\lambda}+B_{\lambda} \operatorname{ch}(\lambda X)\right] \\
& \times \exp \left[-\left(\frac{B o}{4}-\frac{\lambda^{2}}{B o}\right) \theta-\frac{B o}{2} X\right] / C_{\lambda} ; \\
& A_{\lambda}=-(1-K)\left[\frac{1}{2} \operatorname{ch}(\lambda H)+\frac{\lambda}{B o} \operatorname{sh}(\lambda H)\right] \\
& +\left(\frac{1}{2}-K\right) \exp \left(\frac{B o}{2} H\right) \\
& B_{\lambda}=-\frac{1}{B o} \exp \left(\frac{B o}{2} H\right)+(1-K)\left[\frac{1}{2 \lambda} \operatorname{sh}(\lambda H)\right. \\
& \left.+\frac{1}{B o} \operatorname{ch}(\lambda H)\right] \\
& C_{\lambda}=-\left(\frac{B o}{4}-\frac{\lambda^{2}}{B o}\right)\left\{\frac { 1 } { B o } \left[\frac{B o H}{4 \lambda} \operatorname{sh}(\lambda H)+\frac{1}{2 \lambda} \operatorname{sh}(\lambda H)\right.\right. \\
& \left.+\frac{H}{2} \operatorname{ch}(\lambda H)\right]-\left(\frac{1}{2}-K\right)\left[-\frac{B o}{4 \lambda^{3}} \operatorname{sh}(\lambda H)+\frac{B o H}{4 \lambda^{2}} \operatorname{ch}(\lambda H)\right. \\
& \left.\left.+\frac{H}{2 \lambda} \operatorname{sh}(\lambda H)\right]\right\}
\end{aligned}
$$

где $\lambda$ - корень уравнения

$$
\operatorname{th}(\lambda H)=\frac{4 B o K \lambda}{(1-2 K) B o^{2}+4 \lambda^{2}} .
$$

Для минимизации ошибки предположения о параметрическом характере безразмерной текущей высоты слоя использовано балансовое уравнение для частиц

$$
K \int_{0}^{\theta} N(0, \theta) d \theta+\frac{1}{H(\theta)} \int_{0}^{H(\theta)} N(X, \theta) d X=1,
$$

из которого определена корректировка к решению $\Omega(K, B o, \theta)$, равная

$$
\left\{\begin{array}{c}
{\left[P_{\mu} / K+Q_{\mu} / H(\theta)\right]^{-1}, \text { при } 4 K /[(1-2 K) B o]>H,} \\
{\left[\left(P_{\lambda}+P_{\mu}\right) / K+\left(Q_{\lambda}+Q_{\mu}\right) / H(\theta)\right]^{-1}, \text { при } K<1 / 2} \\
\text { и } 4 K /[(1-2 K) B o] \leq H,
\end{array}\right.
$$

$$
P_{\mu}=\sum_{n=1}^{\infty} \frac{B_{\mu}}{\left(\frac{B o}{4}+\frac{\mu_{n}^{2}}{B o}\right)}\left\{1-\exp \left[-\left(\frac{B o}{4}+\frac{\mu_{n}^{2}}{B o}\right) \theta\right]\right\} / C_{\mu}
$$$$
Q_{\mu}=\sum_{n=1}^{\infty}
$$$$
\times\left\langle-\frac{A_{\mu}}{\mu_{n}}\left\{\frac{\left[\frac{B o}{2} \sin \left(\mu_{n} H\right)+\mu_{n} \cos \left(\mu_{n} H\right)\right] \exp \left(-\frac{B o}{2} H\right)-\mu_{n}}{\frac{B o^{2}}{4}+\mu_{n}^{2}}\right\}\right.
$$$$
\left.+B_{\mu}\left\{\frac{\left[\mu_{n} \sin \left(\mu_{n} H\right)-\frac{B o}{2} \cos \left(\mu_{n} H\right)\right] \exp \left(-\frac{B o}{2} H\right)+\frac{B o}{2}}{\frac{B o^{2}}{4}+\mu_{n}^{2}}\right\}\right\rangle
$$$$
\times \exp \left[-\left(\frac{B o}{4}+\frac{\mu_{n}^{2}}{B o}\right) \theta\right] / C_{\mu}
$$$$
P_{\lambda}=\frac{B_{\lambda}}{\left(\frac{B o}{4}-\frac{\lambda^{2}}{B o}\right)}\left\{1-\exp \left[-\left(\frac{B o}{4}-\frac{\lambda^{2}}{B o}\right) \theta\right]\right\} / C_{\mu}
$$$$
Q_{\lambda}=\left\langle\frac{1}{(B o-2 \lambda)}\left(\frac{A_{\lambda}}{\lambda}+B_{\lambda}\right)\left\{1-\exp \left[\left(\lambda-\frac{B o}{2}\right) H\right]\right\}\right.
$$$$
\left.+\frac{1}{(B o-2 \lambda)}\left(\frac{A_{\lambda}}{\lambda}+B_{\lambda}\right)\left\{1-\exp \left[-\left(\lambda+\frac{B o}{2}\right) H\right]\right\}\right\rangle
$$$$
\times \exp \left[-\left(\frac{B o}{4}-\frac{\lambda^{2}}{B o}\right) \theta\right] / C_{\lambda} .
$$

Таким образом, окончательное решение таково:

$$
N(X, \theta)=\Omega(K, B o, \theta) N(X, \theta) .
$$

Оно в точности удовлетворяет начально-краевым условиям, а самому уравнению с точностью

$$
N(X, \theta) \frac{d \operatorname{Ln} \Omega(K, B o, \theta)}{d \theta} \ll 1 .
$$

\section{3. Численное интегрирование}

Оценка точности полученного приближенного аналитического решения проведена численно введением замены

$$
N(X, \theta)=\Phi(X, \theta) \exp \left[-\frac{B o}{2}\left(X+\frac{1}{2} \theta\right)\right]
$$


и новой системы координат [16]

$$
\xi=\frac{X}{1-\alpha \theta}, \quad \eta=\frac{\theta}{1-\alpha \theta},
$$

что позволило получить начально-краевую задачу с фиксированными границами

$$
\begin{gathered}
\frac{\partial \Psi(\xi, \eta)}{\partial \eta}=-\frac{\alpha \xi}{1+\alpha \eta} \frac{\partial \Psi(\xi, \eta)}{\partial \xi}+\frac{1}{B o} \frac{\partial^{2} \Psi(\xi, \eta)}{\partial \xi^{2}} \\
\Psi(\xi, \theta)=\exp \left(\frac{B o}{2} \xi\right) \\
\left(\frac{1}{2}-K\right) \Psi(0, \eta)+\frac{(1+\alpha \eta)}{B o} \frac{\partial \Psi(0, \eta)}{\partial \xi}=0 \\
\frac{1}{2} \Psi(1, \eta)+\frac{(1+\alpha \eta)}{B o} \frac{\partial \Psi(1, \eta)}{\partial \xi}=0 \\
\Psi(\xi, \eta)=\Phi[X(\xi, \eta), \theta(\xi, \eta)]
\end{gathered}
$$

Для системы (9)-(13) использована явная конечноразностная схема $[17,18]$ :

$$
\begin{gathered}
\Psi_{i, j+1}=a_{i, j} \Psi_{i+1, j}+b \Psi_{i, j}+c_{i, j} \Psi_{i-1, j} \\
(i=\overline{1, m-1} ; j=0,1,2, \mathrm{~K}), \\
\Psi_{i, 0}=\exp \left(\frac{B o}{2} i \Delta \xi\right), \\
\Psi_{0, j}=d_{j}\left(4 \Psi_{1, j}-\Psi_{2, j}\right) \quad(j=0,1,2, \mathrm{~K}), \\
\Psi_{m, j}=e_{j}\left(4 \Psi_{m-1, j}-\Psi_{m-2, j}\right) \quad(j=0,1,2, \mathrm{~K}),
\end{gathered}
$$

где

$$
\begin{gathered}
a_{i, j}=\frac{\Delta \eta}{B o \Delta \xi^{2}}-\frac{\alpha i \Delta \eta}{2(1+\alpha j \Delta \eta)} ; \quad b=1-\frac{2 \Delta \eta}{B o \Delta \xi^{2}} \\
c_{i, j}=\frac{\Delta \eta}{B o \Delta \xi^{2}}+\frac{\alpha i \Delta \eta}{2(1+\alpha j \Delta \eta)}, \\
d_{j}=[3-B o \Delta \xi(1-2 K) /(1+\alpha j \Delta \eta)]^{-1} ; \\
e_{j}=[3+B o \Delta \xi /(1+\alpha j \Delta \eta)]^{-1}, \quad \Psi_{i, j}=\Psi\left(\xi_{i}, \eta_{j}\right) \\
\xi_{i}=i \Delta \xi(i=\overline{0, m}) ; \quad \Delta \xi=1 / m ; \\
\eta_{j}=j \Delta \eta(j=0,1,2, \mathrm{~K}) .
\end{gathered}
$$

Расчеты проводились следующим образом: задавались величины $\alpha, B o, K$, выбирался шаг интегрирования из диапазона $\Delta \eta=\left(10^{-5}-10^{-3}\right) \Delta \xi$, где $\Delta \xi=10^{-3}-10^{-1}$, затем в соответствии с вычислительной схемой $(14)-(17)$ определялась сеточная функция $\Psi_{i, j}$, значения которой модифицировались обращением функционального преобразования (8), и производился возврат к исходной безразмерной концентрации по (7). Результаты расчетов при $\alpha=0$ (отсутствует перемещение свободной границы) коррелируют с данными в [14], что подтвердило работоспособность предложенной численной схемы. Для предельного случая, когда скорость движения свободной границы значительно превышает скорость осаждения частиц $(\alpha=1)$, результаты численного интегрирования и вычислений по (6) практически идентичны (рис. 1). Сравнительный анализ показывает, что при затрудненном встраивании частиц в структуру осадка $(K<1)$, неинтенсивное перемешивание $($ Вo > 1$)$ приводит к существенно большему концентрированию частиц вблизи поверхности их осаждения, причем в течение всего процесса локальная концентрация частиц выше в случае перемещения свободной границы. Если поверхность осадка характеризуется интенсивным поглощением частиц $(K>1)$, то процесс осаждения протекает значительно быстрее при движении свободной границы вне зависимости от интенсивности перемешивания в слое.

\section{2. Полидисперсная взвесь}

\section{1. Формулировка и общее решение задачи}

Обобщение модели (1)-(5) на случай малоконцентрированной полидисперсной взвеси проведено с применением принципа суперпозиции концентрационных полей различных фракций частиц в предположении выполнения гипотезы сплошности

$$
\begin{gathered}
\frac{\partial F(X, L, \theta)}{\partial \theta}=\frac{\partial F(X, L, \theta)}{\partial X}+B o^{-1}(L) \frac{\partial^{2} F(X, L, \theta)}{\partial X^{2}}, \\
F(X, L, 0)=F_{0}(L), \\
{[1-K(L)] F(0, L, \theta)+B o^{-1}(L) \frac{\partial F(0, L, \theta)}{\partial X}=0,} \\
F[H(\theta), L, \theta]+B o^{-1}(L) \frac{\partial F[H(\theta), L, \theta]}{\partial X}=0, \\
H(\theta)=1-\alpha \theta,
\end{gathered}
$$

где

$$
\begin{gathered}
F_{0}(L)=\bar{l} f_{0}^{(N)}(l), \quad F(X, L, \theta)=\bar{l} f^{(N)}(x, l, \tau), \quad L=l / \bar{l}, \\
L_{\min }=l_{\min } / \bar{l}, L_{\max }=l_{\max } / \bar{l}, l_{\min }, \quad l_{\max }, \bar{l}=\int_{l_{\min }}^{l_{\max }} l f_{0}^{(N)}(l) d l
\end{gathered}
$$

- минимальный, максимальный и среднечисленный размеры частиц во взвеси $[\mathrm{m}]$;

$$
f_{0}(l), \quad f(x, l, \tau)=\partial n(x, l, \tau) / \partial l
$$

- исходная и локальная счетные функции распределения частиц по размерам $\left[\mathrm{m}^{-4}\right]$;

$$
\begin{gathered}
f_{0}^{(N)}(l)=f_{0}(l) / \int_{l_{\min }}^{l_{\max }} f_{0}(l) d l, \\
f^{(N)}(x, l, \tau)=f(x, l, \tau) / \int_{l_{\min }}^{l_{\max }} f_{0}(l) d l
\end{gathered}
$$

- исходная и локальная нормированные счетные функции распределения частиц по размерам $\left[\mathrm{m}^{-1}\right]$; $\bar{\theta}=\tau[w(\bar{l})+v] / h_{0}$. 

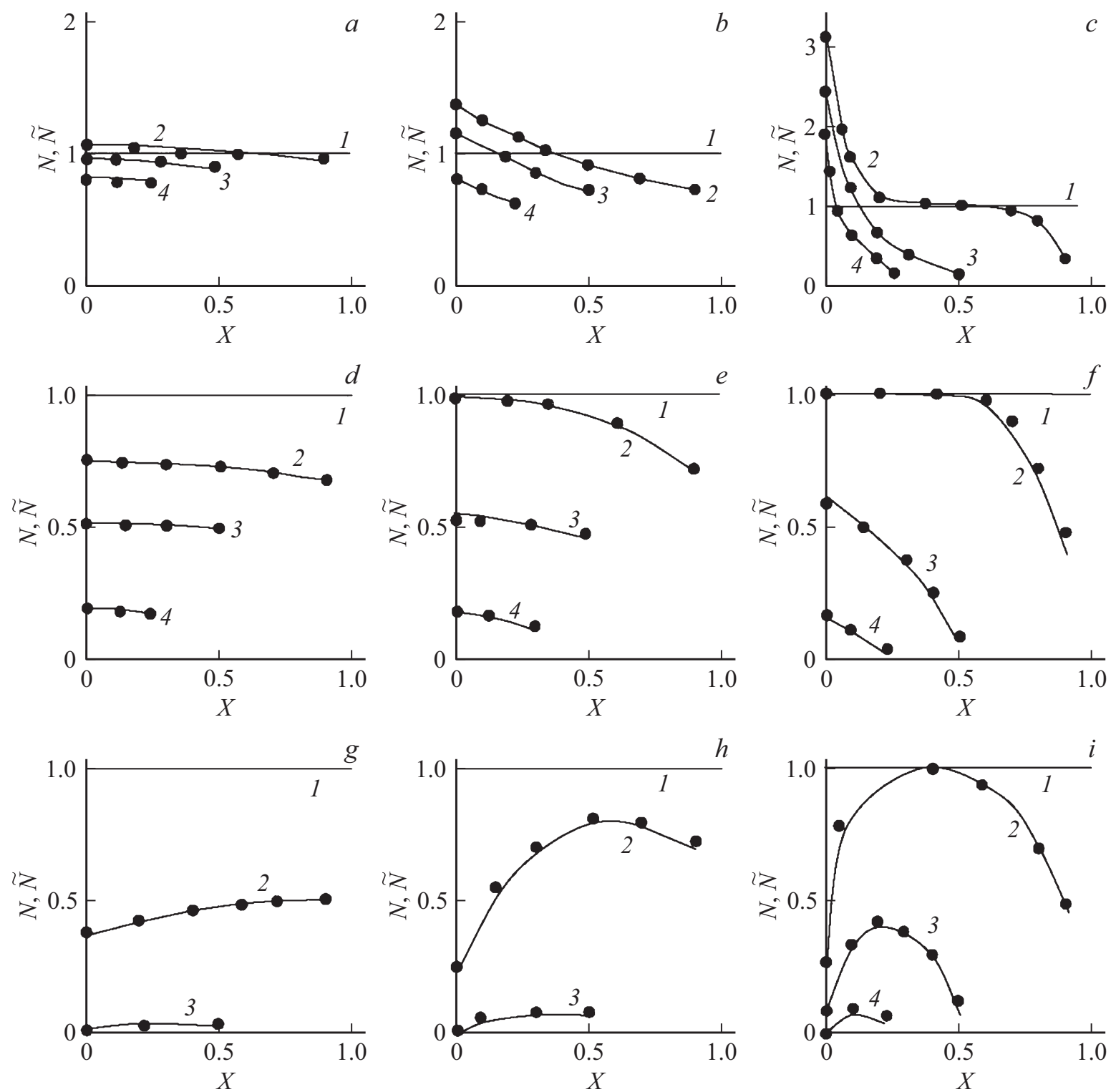

Рис. 1. Безразмерная счетная концентрация монодисперсной взвеси в слое с движущейся свободной границей $(\alpha=1)$ в моменты безразмерного времени $\theta: 1-0,2-0.1,3-0.5,4-0.75$ при различных значениях $K$ и $B o: a-K=0.1, B o=0.1 ; b-K=0.1$, $B o=1 ; c-K=0.1, B o=10 ; d-K=1, B o=0.1 ; e-K=1, B o=1 ; f-K=1, B o=10 ; g-K=10, B o=0.1 ; h-K=10$, $B o=1 ; i-K=10, B o=10$ (линия - расчет по (6); точки - численное интегрирование системы (9)-(13)).

Из сравнения систем уравнений (1)-(5) и (18)-(22) следует, что решение (18)-(22) аналогично (6)

$$
F(X, L, \bar{\theta})=F_{0}(L) \Omega[K(L), B o(L), \bar{\theta}] N(X, L, \bar{\theta}),
$$

где под $\Omega[K(L), B o(L), \bar{\theta}]$ и $N(X, L, \bar{\theta})$ следует понимать выражения для $\Omega(K, B o, \theta)$ и $N(X, \theta)$, в которых учтена зависимость $B o, K$ и $\theta$ от $L$, причем

$$
\theta=\left[\bar{\alpha}+(1-\bar{\alpha}) L^{2}\right] \bar{\theta} .
$$

\section{2. Определение параметров модели}

Согласно [19], перемешивание дисперсной фазы характеризуется конвективным механизмом и броуновской диффузией

$$
D=D_{c}+D_{b},
$$

причем, согласно [20]:

$$
D_{c}=\varepsilon_{c} D_{b}, \quad D_{b}=\frac{k_{\mathrm{B}} T}{2 \pi l \nu \rho},
$$

поэтому

$$
D(l)=\left(1+\varepsilon_{c}\right) \frac{k_{\mathrm{B}} T}{2 \pi l \nu \rho},
$$

где $k_{\mathrm{B}}-$ постоянная Больцмана $[\mathrm{J} / \mathrm{K}], \varepsilon_{c}-$ коэффициент конвекции, $\varepsilon-$ порозность осадка взвеси, $v-$ коэффициент кинематической вязкости несущей среды $\left[\mathrm{m}^{2} / \mathrm{s}\right]$. 
Кинетический коэффициент $k$ определен по аналогии c $[13,21]:$ приблизившись к поверхности осаждения, частицы взвеси утрачивают конвективную составляющую движения, но сохраняют броуновскую и седиментационную, т.е.

$$
k(l)=\frac{k_{0}(l)}{1+\left[k_{0}(l)-w(l)-v\right] / u_{V}},
$$

где

$$
\begin{aligned}
& k_{0}(l)= {\left[2 \pi / \bar{v}_{b}^{2}(l)\right] \exp \left\{-\frac{1}{2}[w(l)+v] / \bar{v}_{b}^{2}(l)\right\} } \\
&+ \frac{1}{2}[w(l)+v]\left\langle 1+\operatorname{erf}\left\{[w(l)+v] /\left[2 \bar{v}_{b}^{2}(l)\right]^{1 / 2}\right\}\right\rangle, \\
& \bar{v}_{b}^{2}(l)=\frac{6 k_{\mathrm{B}} T}{\pi l^{3} \rho}, u_{V}=\frac{D(l)}{h_{0}}, w(l)=\frac{g\left(\rho_{T}-\rho\right)}{18 \rho v} l^{2},
\end{aligned}
$$

где $g$ - ускорение свободного падения $\left[\mathrm{m} / \mathrm{s}^{2}\right] \rho, \rho_{T}-$ плотность несущей среды и частиц взвеси $\left[\mathrm{kg} / \mathrm{m}^{3}\right]$.

Параметры модели (24) и (25) представлены в относительном виде через их значения для среднечисленного размера частиц

$$
\begin{gathered}
B o(L)=L\left[\bar{\alpha}+(1-\bar{\alpha}) L^{2}\right] \overline{B o}, \\
K(L)=\frac{Q(L, \chi)}{1+[Q(L, \chi)-1]\left[\bar{\alpha}+(1-\bar{\alpha}) L^{2}\right] L \overline{B o}},
\end{gathered}
$$$$
Q(L, \chi)=\frac{(1-\bar{\alpha}) \chi^{-1} L^{-\frac{7}{2}}}{\sqrt{2 \pi}\left[\bar{\alpha}+(1-\bar{\alpha}) L^{2}\right]} \exp \left\{-\frac{\left[\bar{\alpha}+(1-\bar{\alpha}) L^{2}\right]^{2}}{2(1-\bar{\alpha})^{2}} \chi^{2} L^{7}\right\}
$$$$
+\frac{1}{2}\left\langle 1+\operatorname{erf}\left\{\frac{\left[\bar{\alpha}+(1-\bar{\alpha}) L^{2}\right]}{2(1-\bar{\alpha})} \chi L^{\frac{7}{2}}\right\}\right\rangle,
$$

$$
\chi=\left(\bar{l} / l^{*}\right)^{7 / 2}, \quad l^{*}=\left(\frac{6 k_{\mathrm{B}} T}{\pi \rho}\right)^{\frac{1}{7}} /\left[\frac{g\left(\rho_{T}-\rho\right)}{18 \rho v}\right]^{\frac{2}{7}},
$$

где $\bar{\alpha}=v /[w(\bar{l})+v], \overline{B o}=[w(\bar{l})+v] h_{0} / D(\bar{l})$ - число Боденштейна, $T$ - температура взвеси $[\mathrm{K}]$.

Для стоксовских частиц с увеличением $L K(L) \rightarrow 1$, поэтому параметр $\chi$, по существу, является мерой отклонения частиц размера $\bar{l}$ от броуновских (рис. 2 ).

\section{3. Вычислительный эксперимент}

Расчеты проводились для исходной функции плотности распределения частиц по размерам в виде $F_{0}(L)=\exp (-L)$ в диапазоне изменения $L$ от $L_{\min }=0.01$ до $L_{\max }=3$, что отвечало условию нормировки с точностью до 5\%. Вычисления осуществлялись при числе характеристических корней $n$ в решении (23), равным 100 , что обеспечивало удовлетворительную сходимость.

В случае интенсивного $(\overline{B o} \ll 1)$ перемешивания функции плотности распределения частиц по размерам на свободной поверхности и у дна практически идентичны (рис. 3, $a, b$ ), причем уменьшение высоты несущей среды в слое увеличивает общее число частиц в объеме

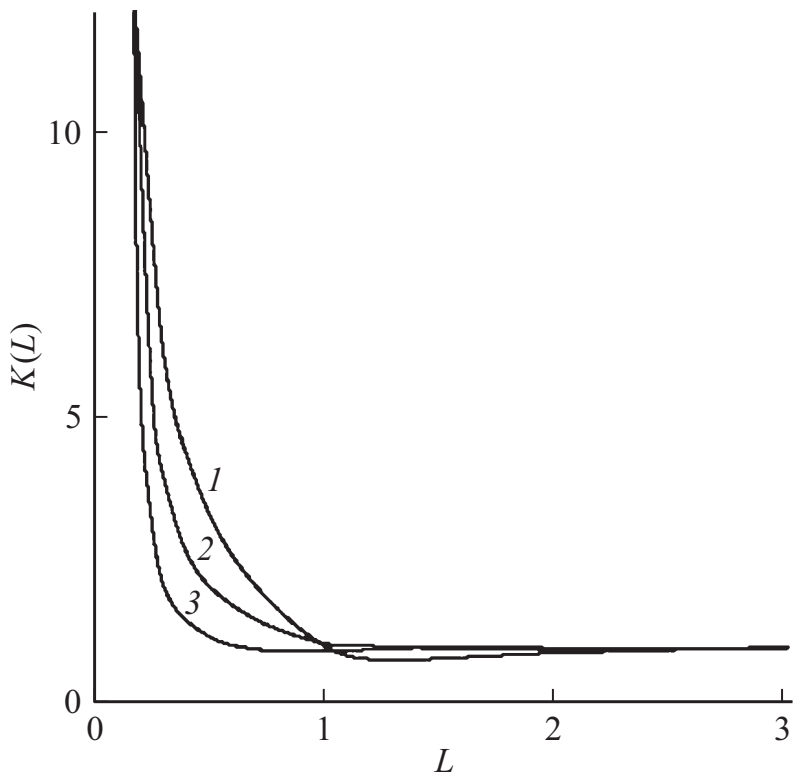

Рис. 2. Зависимость $K(L)$ при $\overline{B o}=1$ и различных значениях $\chi: 1-0.1,2-1,3-10$.

с дрейфом их максимума в область „мелких“ фракций, как и большее количество стоксовских частиц во взвеси $\chi \gg 1$. При $\overline{B o} \gg 1$ (рис. $3, c, d$ ) наблюдается существенное различие между функциями распределения на дне и свободной поверхности, характеризующих сепарацию частиц в процессе осаждения с сохранением тех же закономерностей, что и при $\overline{B o} \ll 1$.

Для практических целей локальная относительная массовая концентрация является более информативной:

$C(X, \bar{\theta})=\frac{c(x, \tau)}{c_{0}}=\int_{L_{\min }}^{L_{\max }} L^{3} F(X, L, \bar{\theta}) d L / \int_{L_{\min }}^{L_{\max }} L^{3} F_{0}(L) d L$,

где

$c_{0}=k_{V} \rho_{T} \int_{l_{\min }}^{l_{\max }} l^{3} f_{0}(l) d l, \quad c(x, \tau)=k_{V} \rho_{T} \int_{l_{\min }}^{l_{\max }} l^{3} f(x, l, \tau) d l$

- исходная и локальная массовые концентрации частиц во взвеси $\left[\mathrm{kg} / \mathrm{m}^{3}\right]$.

Вычисления показали, что в случае исходной экспоненциальной функции плотности распределения частиц по размерам, наиболее существенное влияние на профиль концентрации по высоте слоя оказывает интенсивность перемешивания (рис. 4). При этом во всех случаях движение свободной границы вместе с несущей средой к поверхности осаждения приводит к увеличению относительной массовой концентрации по сравнению с покоящейся свободной границей. Дополнительный анализ скорости роста осадка показал, что наибольшая скорость осаждения полидисперсной взвеси при движении свободной границы наблюдается вблизи 

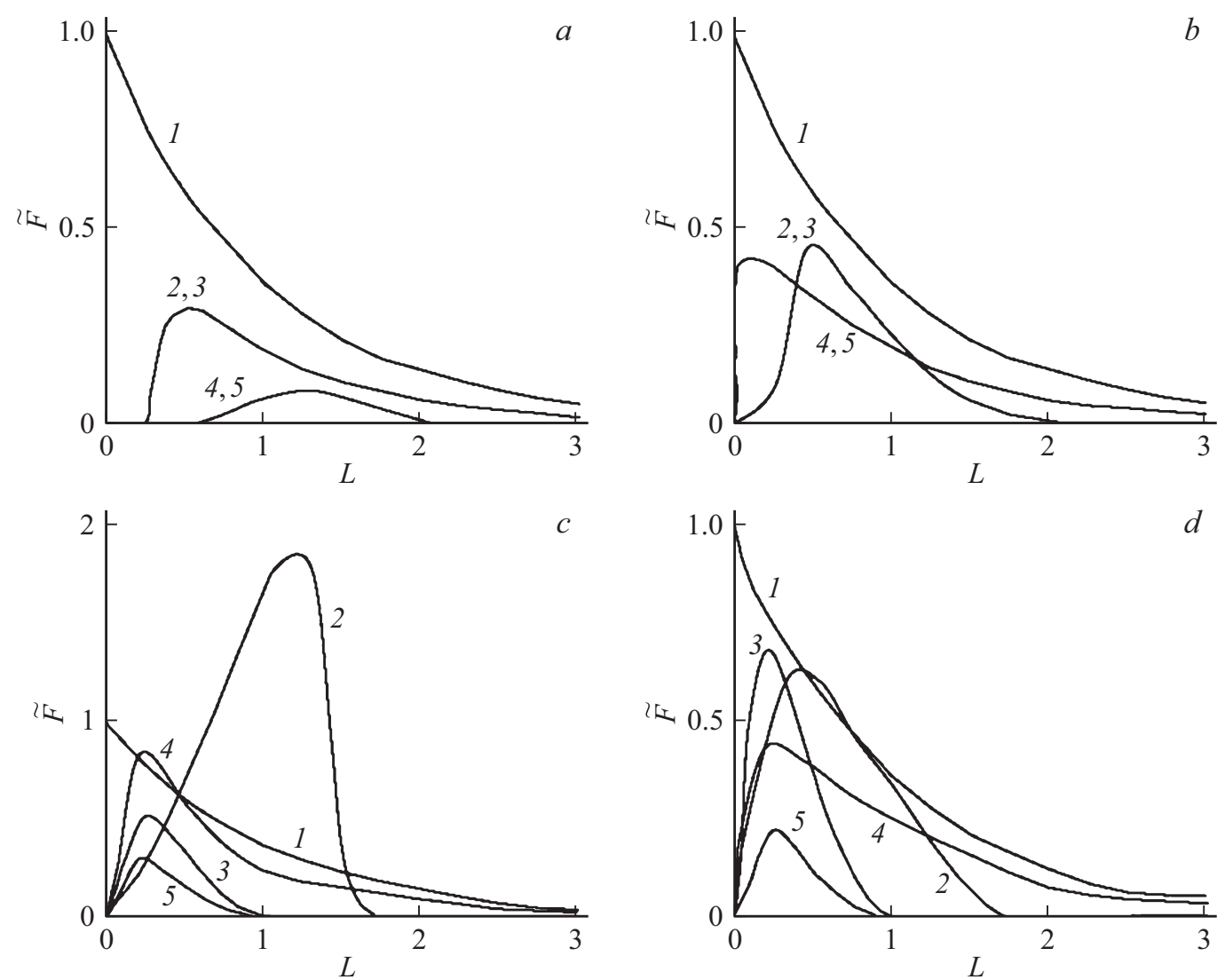

Рис. 3. Локальные функции распределения частиц по размерам в момент безразмерного времени $\theta=0.5 \mathrm{c} F_{0}(L)=\exp (-L)$ (кривая 1$)$ при $\alpha=0(2-X=0 ; 3-X=1)$ и $\alpha=1(4-X=0 ; 5-X=0.5)$ для различных вариантов $\overline{B o}$ и $\chi: a-0.1$ и 0.1 ; $b-0.1$ и $10 ; c-10$ и $0.1 ; d-10$ и 10.
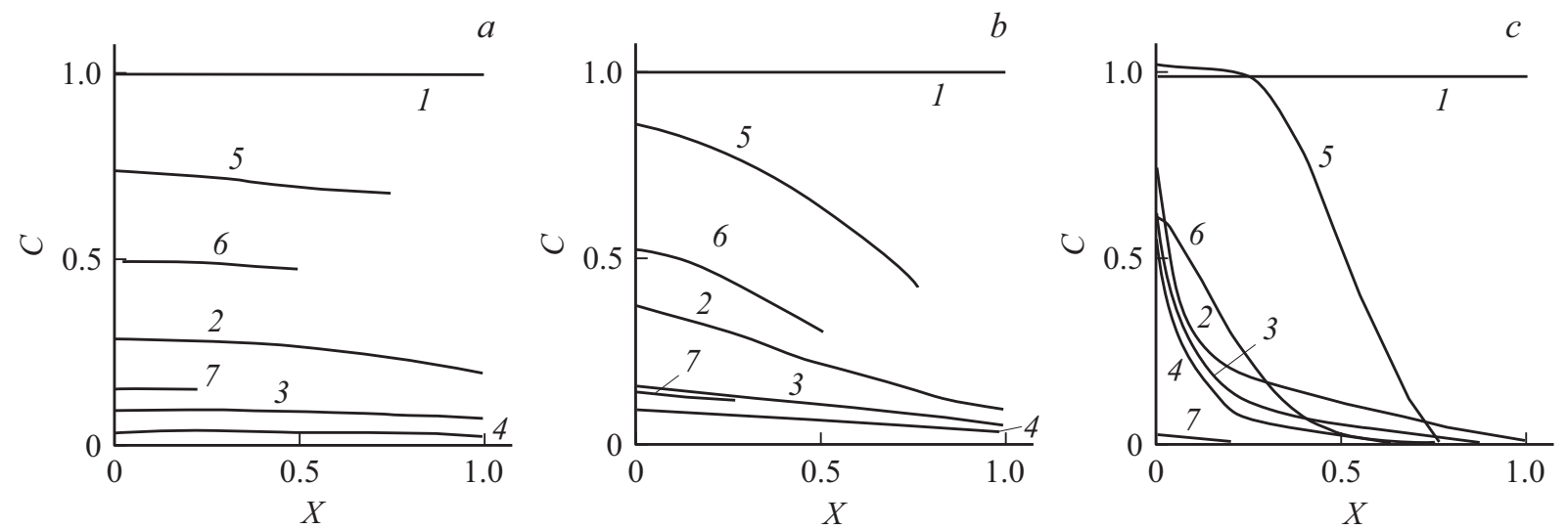

Рис. 4. Относительная массовая концентрация полидисперсной взвеси с $F_{0}(L)=\exp (-L)$ при различных $\overline{B o}: a-0.1 ; b-1 ;$ $c-10$ в момент безразмерного времени $\bar{\theta}$ для $\alpha=0: 1-0 ; 2-0.25 ; 3-0.5 ; 4-0.75$ и $\alpha=1: 1-0 ; 5-0.25 ; 6-0.5$; $7-0.7$.

значения $\overline{B o} \approx 1$, т.е. когда скорость седиментации частиц среднечисленного размера сравнима со скоростью конвективного переноса в дисперсионной фазе:

где

$$
\begin{gathered}
\sigma(\bar{\theta})=\delta(\tau) / \delta_{0}, \quad \delta_{0}=\frac{k_{V}}{1-\varepsilon} h_{0} \int_{l_{\min }}^{l_{\max }} l^{3} f_{0}(l) d l, \\
\delta(\tau)=\frac{k_{V}}{1-\varepsilon} \int_{l_{\min }}^{l_{\max }} l^{3} k(l)\left[\int_{0}^{\tau} f(0, l, \tau) d \tau\right] d l
\end{gathered}
$$$$
\frac{d \sigma(\bar{\theta})}{d \bar{\theta}}=\int_{L_{\min }}^{L_{\max }} L^{5} K(L) F(0, L, \bar{\theta}) d L / \int_{L_{\min }}^{L_{\max }} L^{3} F_{0}(L) d L,
$$ 


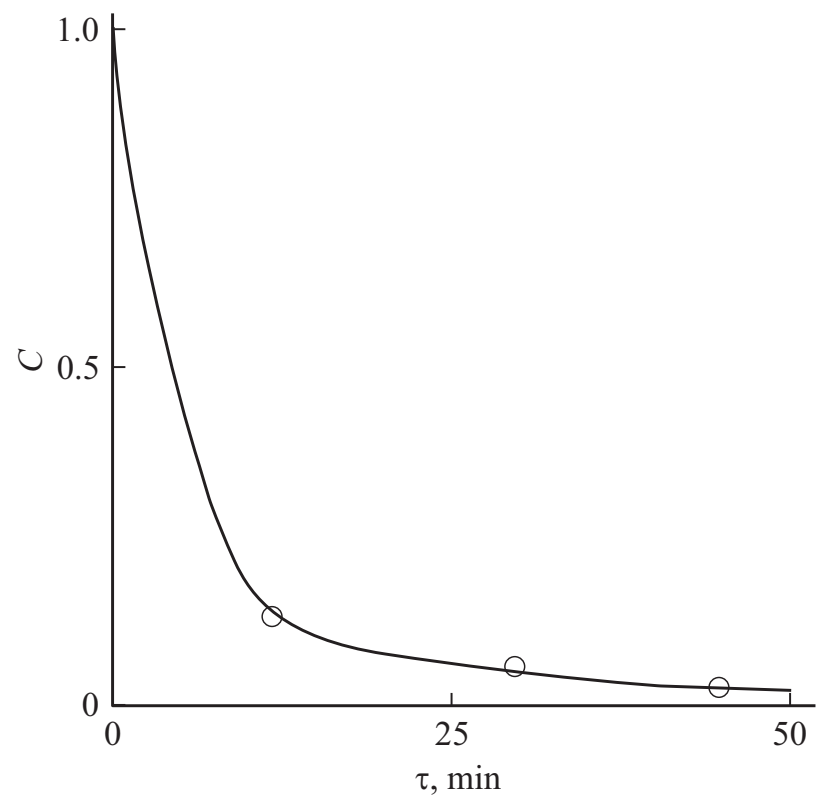

Рис. 5. Изменение концентрации кристаллического азота в жидком водороде для условий опыта в точке отбора: - - расчет по модели; о — эксперимент [22].

- толщина осадка частиц взвеси при полном их осаждении и текущая толщина осадка $[\mathrm{m}]$.

Для оценки корректности модельных представлений и адекватности полученных результатов расчетов проведено сравнение с экспериментальными данными по кинетике осаждения кристаллического азота в жидком водороде при теплопритоке $70 \mathrm{~W} / \mathrm{m}^{2}$ через стенки опытного криогенного резервуара [22]. Охлаждение насыщенного растворенным азотом жидкого водорода с образованием, ростом и седиментацией отвержденного азота достигалось вакуумированием парового пространства. Седиментометрический анализ и фиксация изменения массовой концентрации взвеси кристаллического азота проводились на глубине $0.28 \mathrm{~m}$ при высоте столба жидкости $0.52 \mathrm{~m}$ соответственно электроемкостным датчиком и пробоотборником с последующей подачей криосуспензии через газификационную камеру на хроматограф. Было установлено, что счетная функция плотности распределения кристаллов азота по размерам близка к экспоненциальному виду со средним размером $\approx 1.3 \mu \mathrm{m}$. Поскольку объемный расход газифицированной криосуспензии составлял $4 \mathrm{~m}^{3} / \mathrm{h}$, а внутренний диаметр резервуара был $0.63 \mathrm{~m}$, скорость движения свободной поверхности жидкого водорода есть $4.68 \cdot 10^{-6} \mathrm{~m} / \mathrm{s}$. С учетом того что стоксовская скорость осаждения среднечисленного размера кристаллического азота равна $7.59 \cdot 10^{-5} \mathrm{~m} / \mathrm{s}, \bar{\alpha}=0.058$. Исходя из полученной оценки коэффициента конвективной диффузии жидкого водорода, равного $1.33 \cdot 10^{-5} \mathrm{~m}^{2} / \mathrm{s}$, найдено значение числа Боденштейна для среднечисленного размера кристаллов азота $\overline{B o}=3.15$, а также $\chi=3.15$. Результаты сравнения приведены на рис. 5.

\section{Заключение}

Предложенная на основе конвективно-диффузионного подхода к переносу дисперсной фазы в малоконцентрированных взвесях в условиях перемешивания математическая модель корректным образом качественно и количественно описывает динамику изменения концентрационных полей и кинетику образования осадка при движении свободной границы.

\section{Список литературы}

[1] Таубман Е.И. Выпаривание (процессы и аппараты химической и нефтехимической технологии). М.: Химия, 1982. $328 \mathrm{c}$.

[2] Ельшин А.И. Теоретические и технологические аспекты разделения дисперсных систем фильтрованием. Новополоцк: ПГУ, 1994. 96 с.

[3] Flynn M.T. Cryogenic Engineering. NY: Marcel Dekker, 2005. 873 p.

[4] Crank J. Free and Moving Boundary Problems. Oxford: Clarendon Press, 1984. 425 p.

[5] Tarzia D.A. // MAT. Serie A. 2000. N 2. P. 297-301.

[6] Нигматулин Р.Н. Основы механики гетерогенных сред. М.: Наука, 1978. 336 с.

[7] Morel C. Mathematical Modeling of Disperse Two-Phase Flows. NY:: Springer, 2015. 350 p. DOI: DOI:10.1007/9783-319-20104-7

[8] Броунштейн В.Б. // ЖПХ. 1983. Т. 56. № 8. С. 1788-1793.

[9] Ungarish M. Hydrodynamics of Suspensions: Fundamental of Centrifugal and Gravity Separation. NY:: Springer, 1993. $325 \mathrm{p}$.

[10] Веригин А.Н., Васильев С.В. // Теор. основы хим. технол. 1982. T. 16. № 3. C. 374-380.

[11] Ряжских А.В., Ряжских В.И., Богер А.А., Слюсарев М.И. // ИФЖ. 2013. Т. 86. № 6. С. 1206 1211. [Ryazhskikh A.V., Ryazhskikh V.I., Boger A.A., Slyusarev M.I. // J. Eng. Phys. Thermophys. 2013. Vol. 86. N 6. P. $1280-1285$.

[12] Кондратьев А.С., Швыдько П.П. // Теор. основы хим. технол. 2017. Т. 51. № 1. С. 99-110. [Kondat'ev A.S., Shvyd'ko P.P. // Theor. Found. Chem. Eng. 2017. Vol. 51. N 1. P. 107-117. DOI: 10.1134/S0040579517010122]

[13] Харин В.М., Ряжских В.И., Завадских Р.М. // Теор. основы хим. технол. 1996. Т. 30. № 5. С. 453-457. [Kharin V.M., Ryazhskikh V.I., Zavadskikh R.M. // Theor. Found. Chem. Eng. 1996. Vol. 30. N 5. P. 409-413.]

[14] Харин В.М., Ряжских В.И. // Теор. основы хим. технол. 1989. T. 23. № 5. C. 651-658. [Kharin V.M., Ryazhskikh V.I. // Theor. Found. Chem. Eng. 1990. Vol. 23. N 5. P. 494-501.]

[15] Деч $Г$. Руководство к практическому применению преобразования Лапласа и z-преобразования. М.: Физматгиз, 1971. $288 \mathrm{c}$.

[16] Joos P. Dynamic Surface Phenomena. AHZeist: VSP BP, 1999. 360 p.

[17] Самарский А.А., Вабищевич П.Н. Вычислительная теплопередача. М.: Едиториал УРСС, 2003. 785 с.

[18] Березин И.С., Жидков Н.П. Методы вычислений. В 2-ч т. Т. 1. М.: Физматлит, 1959. 464 с. 
[19] Берд Р., Стьюарт В., Лайтфут Е. Явления переноса. М.: Химия, 1974. $688 \mathrm{c.}$

[20] Ландау Л.Д., Лифиии, Е.М. Теоретическая физика. В 10 т. T. VI. Гидродинамика. М.: Наука, 1982. 736 с.

[21] Седунов Ю.С. // Изв. АН СССР. Сер. геофиз. 1964. № 7. C. $1093-1102$.

[22] Харин В.М., Ряэских В.И., Завадских Р.М. // Теор. основы хим. технол. 1991. Т. 25. № 5. С. 659-669. [Kharin V.M., Ryazhskikh V.I. // Theor. Found. Chem. Eng. 1991. Vol. 25. N 5. P. 659-669.] 